\title{
Glucose uptake in human brown adipose tissue is impaired upon fasting-induced insulin resistance
}

\author{
Mark J. W. Hanssen • Roel Wierts • Joris Hoeks • \\ Anne Gemmink • Boudewijn Brans • Felix M. Mottaghy • \\ Patrick Schrauwen • Wouter D. van Marken Lichtenbelt
}

Received: 25 October 2014 / Accepted: 17 November 2014 / Published online: 13 December 2014

(C) Springer-Verlag Berlin Heidelberg 2014

\begin{abstract}
Aims/hypothesis Human brown adipose tissue (BAT) has recently emerged as a potential target in the treatment of type 2 diabetes, owing to its capacity to actively clear glucose from the circulation - at least upon cold exposure. The effects of insulin resistance on the capacity of human BAT to take up glucose are unknown. Prolonged fasting is known to induce insulin resistance in peripheral tissues in order to spare glucose for the brain.

Methods We studied the effect of fasting-induced insulin resistance on the capacity of BAT to take up glucose during cold exposure as well as on cold-stimulated thermogenesis. BAT glucose uptake was assessed by means of cold-stimulated dynamic 2-deoxy-2-[ $\left.{ }^{18} \mathrm{~F}\right]$ fluoro-D-glucose positron emission tomography/computed tomography $\left(\left[{ }^{18} \mathrm{~F}\right] \mathrm{FDG}-\mathrm{PET} / \mathrm{CT}\right)$ imaging.
\end{abstract}

Electronic supplementary material The online version of this article (doi:10.1007/s00125-014-3465-8) contains peer-reviewed but unedited supplementary material, which is available to authorised users.

M. J. W. Hanssen · J. Hoeks $・$ P. Schrauwen ·

W. D. van Marken Lichtenbelt $(\square)$

Department of Human Biology, NUTRIM School for Nutrition, Toxicology and Metabolism, Maastricht University Medical Centre+ (MUMC+), P.O. Box 616, 6200 MD Maastricht, the Netherlands

e-mail: markenlichtenbelt@maastrichtuniversity.nl

R. Wierts $\cdot$ B. Brans $\cdot$ F. M. Mottaghy

Department of Nuclear Medicine, Maastricht University Medical

Centre+ $(\mathrm{MUMC}+)$, Maastricht, the Netherlands

\section{A. Gemmink}

Department of Movement Sciences, NUTRIM School for Nutrition, Toxicology and Metabolism, Maastricht University Medical Centre+ (MUMC+), Maastricht, the Netherlands

F. M. Mottaghy

Department of Nuclear Medicine, University Hospital RWTH

Aachen, Aachen, Germany
Results We show that a $54 \mathrm{~h}$ fasting period markedly decreases both cold-induced BAT glucose uptake and nonshivering thermogenesis (NST) during cold stimulation. In vivo molecular imaging and modelling revealed that the reduction of glucose uptake in BAT was due to impaired cellular glucose uptake and not due to decreased supply. Interestingly, decreased BAT glucose uptake upon fasting was related to a decrease in core temperature during cold exposure, pointing towards a role for BAT in maintaining normothermia in humans.

Conclusions/interpretation Cold-stimulated glucose uptake in BAT is strongly reduced upon prolonged fasting. When cold-stimulated glucose uptake in BAT is also reduced under other insulin-resistant states, such as diabetes, cold-induced activation of BAT may not be a valid way to improve glucose clearance by BAT under such conditions.

Trial registration: www.trialregister.nl NTR3523

Funding: This work was supported by the EU FP7 project DIABAT (HEALTH-F2-2011-278373 to WDvML) and by the Netherlands Organization for Scientific Research (TOP 91209037 to WDvML).

Keywords Brown adipose tissue $\cdot$ Insulin resistance

\begin{tabular}{ll}
\multicolumn{2}{l}{ Abbreviations } \\
BAT & Brown adipose tissue \\
BMR & Basal metabolic rate \\
{$\left[{ }^{18} \mathrm{~F}\right]$ FDG } & 2-deoxy-2- $\left[{ }^{18} \mathrm{~F}\right]$ fluoro-D-glucose \\
NST & Nonshivering thermogenesis \\
PET/CT & $\begin{array}{l}\text { Positron emission tomography/computed } \\
\text { tomography }\end{array}$ \\
RQ & $\begin{array}{l}\text { Respiratory quotient } \\
\text { SUV }\end{array}$ \\
Standardised uptake value
\end{tabular}




\section{Introduction}

Brown adipose tissue (BAT) is currently regarded as a potential target in the prevention and treatment of obesity and type 2 diabetes. BAT in animals appears to have a high metabolic capacity and is able to actively clear glucose from the circulation $[1,2]$. It is now well recognised that BAT is present and active in human adults [3,4] and that its oxidative metabolism contributes to nonshivering thermogenesis (NST) during cold exposure [5].

Several rodent studies have shown that obese diabetic rodents have an impaired cold tolerance [6-9] associated with a reduced capacity for NST [6] and reduced BAT activity $[8,9]$. Some of these studies indicated that not obesity per se, but also insulin resistance contributes significantly to cold intolerance and reduced BAT activity $[8,10,11]$. In humans, several studies have recently shown negative correlations between BAT activity and adiposity [3, 12, 13] and age [14]. A negative association between BAT activity and diabetic status, independent of BMI and age, has also been reported in a retrospective study [15]. In addition, an impaired thermogenic response to mild cold has been found in diabetic women compared with obese women [16], suggesting a role for insulin resistance in mediating these impairments. However, a confounding factor in these studies is that both obesity and ageing are characterised by reduced BAT activity, independent of diabetes status.

To address whether insulin resistance is directly involved in reducing BAT activity and NST, a 48 to $60 \mathrm{~h}$ fasting period can be employed to induce insulin resistance in young, healthy individuals [17-19]. We have recently shown that this prolonged fasting leads to a $50 \%$ reduction in insulin sensitivity, as determined by the gold standard method, hyperinsulinaemic-euglycaemic clamp, probably due to increased NEFA levels and/or accumulation of triacylglycerols in peripheral tissues [18]. As a result, glucose uptake is reduced in peripheral tissues - mainly skeletal muscle, which is responsible for $\sim 80 \%$ of glucose uptake under insulinstimulated conditions, such as during a hyperinsulinaemiceuglycaemic clamp [20]. The physiological relevance of this peripheral insulin resistance is to spare glucose as a substrate for the brain. However, whether the capacity to stimulate glucose uptake into BAT is also reduced under insulinresistant conditions is presently unknown. Since BAT relies on both carbohydrate and lipid oxidation for its heat production [21], an impaired glucose uptake under fasting-induced insulin-resistant conditions would hamper its thermogenic capacity and could lead to reduced capacity to defend body temperature upon cold exposure. On the other hand, if glucose uptake into BAT could be stimulated by cold exposure under such insulin-resistant conditions, activation of glucose uptake into BAT by cold exposure or other means could serve as an interesting target to increase glucose clearance during insulin resistance.

To evaluate whether glucose uptake into BAT is impaired during fasting-induced insulin resistance, we here examined 16 young, healthy participants in which BAT glucose uptake was measured after both baseline and $54 \mathrm{~h}$ fasting conditions using dynamic 2-deoxy-2- $\left[{ }^{18} \mathrm{~F}\right]$ fluoro-D-glucose positron emission tomography/computed tomography $\left(\left[{ }^{18} \mathrm{~F}\right] \mathrm{FDG}-\right.$ $\mathrm{PET} / \mathrm{CT}$ ) imaging under cold-stimulated conditions, in the absence of shivering [22].

\section{Methods}

Participants Eight male and eight female participants (age $21.9 \pm 3.1$ years; BMI $21.3 \pm 1.5, \mathrm{~kg} / \mathrm{m}^{2}$; fat percentage $20.8 \pm$ $7.8 \%$ ) were included in the study. All participants were screened for medical history and status. Studies were performed between August 2012 and June 2013. All females were on oral contraceptives (ethinyl estradiol or levonorgestrel) and were not measured during their menstruation period. Exclusion criteria included diabetes mellitus, pregnancy, physical activity more than twice per week, history of cardiovascular diseases and asthma or other obstructive pulmonary diseases.

Study design Participants took part in two experimental protocols: a baseline condition and a fasted condition, in a randomised crossover design. These conditions both included a similar experimental day. In the fasted condition, this experimental day was preceded by a $48 \mathrm{~h}$ fasting period. A standardised evening meal was prescribed the day before the start of each experimental protocol.

For the baseline condition (Fig. 1a), consisting of the experimental day only, participants came in at 07:00 hours after an overnight fast and were asked to refrain from heavy exercise $48 \mathrm{~h}$ before the measurements. After arrival, a muscle biopsy was taken from the vastus lateralis muscle [23], and a light breakfast (energy content $<10 \%$ of basal metabolic rate [BMR]) was given subsequently. Body composition was determined by means of dual x-ray absorptiometry (Discovery A, Hologic, Bedford, MA, USA). After a $4 \mathrm{~h}$ fasting period, an individualised cooling protocol was performed at 11:45 hours followed by $\left[{ }^{18} \mathrm{~F}\right] \mathrm{FDG}-\mathrm{PET} / \mathrm{CT}$ scanning (Gemini TF PETCT, Philips, Eindhoven, the Netherlands) at 14:30 hours. For this purpose, a cannula was inserted in the left antecubital vein for blood sampling during thermoneutral and mild cold conditions and injection of the tracer. Participants swallowed a telemetric pill (CoreTemp HT150002, HQ, Palmetto, FL, USA), and iButtons (Maxim Integrated Products, San Jose, CA, USA) were placed on 14 ISO-defined sites [24] for measurements of core and skin temperatures, respectively. A chest strap (Polar T31; Polar, Kempele, Finland) was attached 
Fig. 1 Study protocols for (a) the baseline condition and (b) the fasted condition a

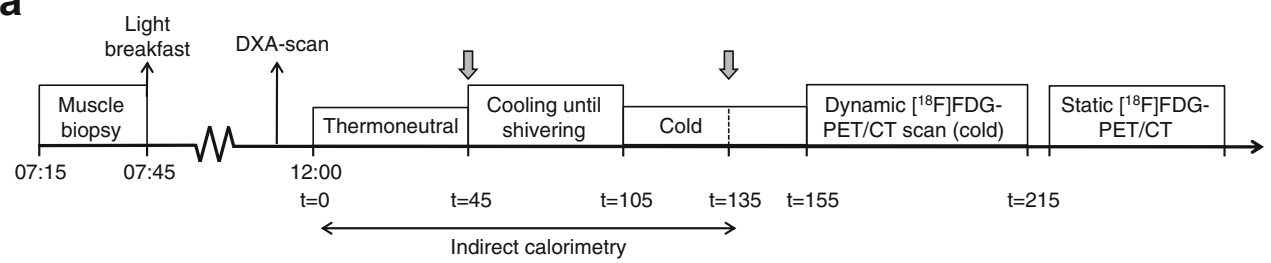

b

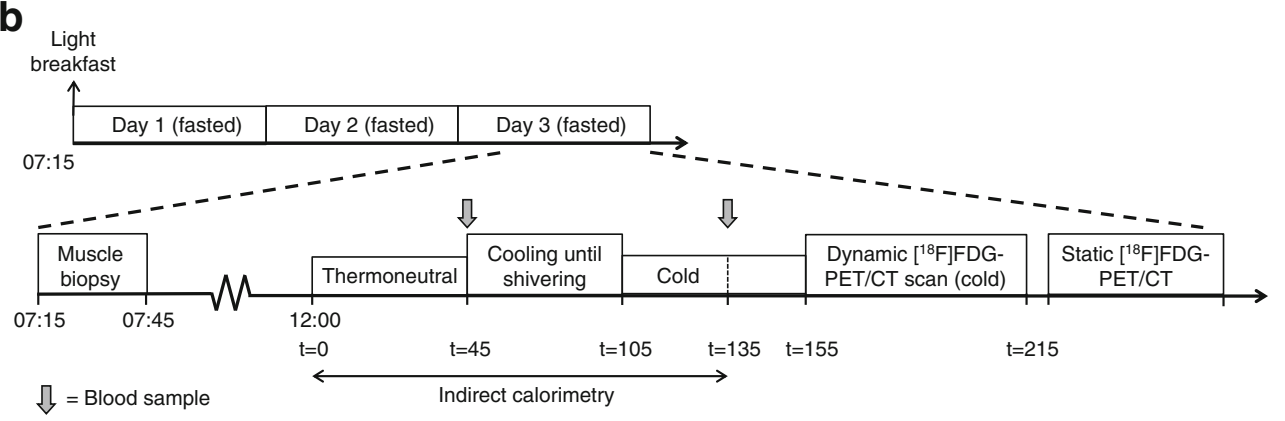

for measurement of heart rate and a pressure cuff (MTP, Medisana, Kerkrade, the Netherlands) was placed on the left arm for measurement of blood pressure. Laser Doppler probes were attached for skin perfusion measurements at the ventral sides of the hand, forearm and hallux, and at the abdomen halfway between the umbilicus and the left lateral side of the body (PF5000, Perimed, Järfälla, Sweden) [22]. Shivering intensity was monitored by electromyography (EMG) sensors (PASAQ, IDEE, Maastricht, the Netherlands) placed at vastus lateralis, pectoralis major and latissimus dorsi muscle [5]. A face-mask was used for continuous measurement of energy expenditure (EE) by means of indirect calorimetry (Omnical, IDEE).

For the fasted condition (Fig. 1b), participants came in at 07:00 hours on day 1 after an overnight fast and were given a light breakfast (energy content $<10 \%$ of BMR). Subsequently, participants stayed in a controlled room in our research unit for $48 \mathrm{~h}$ to ensure compliance to the fasting regime (no solid foods, calorie-free drinks only). They were given the opportunity for supervised light physical exercise (walking, stretching) for 30-60 $\mathrm{min} /$ day. On day 3, a muscle biopsy was taken in the morning at 07:15 hours, and the cooling protocol and $\left[{ }^{18} \mathrm{~F}\right] \mathrm{FDG}-\mathrm{PET} / \mathrm{CT}$ scanning was performed in the afternoon, similar to the experimental day during the baseline condition.

Individualised cooling protocol and PET/CT scanning protocol The cooling and PET/CT scanning protocol was performed as described previously [22]. Briefly, participants were wrapped in a water-perfused suit (ThermaWrap Universal 3166, MTRE Advanced Technologies, Yavne, Israel) and were measured at thermoneutrality for $45 \mathrm{~min}$. Hereafter, participants were cooled until a temperature just above their shivering point and measured for $30 \mathrm{~min}$ at this maximal NST temperature; NST was calculated as the percentage increase in energy expenditure above BMR (measured at thermoneutrality) at a temperature just above the individual's shivering temperature. Subsequently, participants were brought to the PET/CT scanner where imaging started with a low dose CT scan (120 kV, $30 \mathrm{mAs})$. Participants were then injected with $74 \mathrm{MBq}$ of $\left[{ }^{18} \mathrm{~F}\right] \mathrm{FDG}$ at the start of the PET scanning protocol. Both a dynamic [22] and static [3] scan (six to seven bed positions, 6 min per bed position) were acquired as described previously.

PET analysis PET/CT scans were analysed using PMOD software (version 3.0, PMOD technologies, Zurich, Switzerland). Both the researcher (MJW Hanssen) and an experienced nuclear medicine physician (B. Brans) interpreted the PET/CT images. Glucose uptake rates in BAT were determined from the dynamic PET data, as described previously [22]. Two-tissue compartment modelling was employed to iteratively derive values for the individual rate constants $K_{1}$ to $k_{4}$ using a nonlinear least squares method [25], whereby $k_{4}$ was fixed at zero (Electronic Supplementary Material [ESM] Fig. 1).

For determination of mean and maximal $\left[{ }^{18} \mathrm{~F}\right] \mathrm{FDG}$ uptake in BAT, expressed as standardised uptake values (SUV): $\left(\left[{ }^{18} \mathrm{~F}\right] \mathrm{FDG}\right.$ uptake $\left.[\mathrm{kBq} / \mathrm{ml}]\right) /($ injected dose $[\mathrm{kBq}] /$ patient weight $[\mathrm{g}]$ ), regions of interest were manually outlined in the static PET scan [26]. A threshold of 1.5 SUV and Hounsfield units between -10 and -180 were used to define BAT, as described previously [26].

Fixed volumes of interest were selected in supraclavicular BAT, subcutaneous and visceral white adipose tissue (WAT), skeletal muscle (SM), liver and brain to compare $\left[{ }^{18} \mathrm{~F}\right] \mathrm{FDG}$ uptake (calculated as SUV mean) among these tissues, as described previously [27]. 
Skeletal muscle mitochondrial isolation and respiration Skeletal muscle mitochondria were isolated as described previously [22], with minor modifications. Briefly, a portion ( 200 mg) of the muscle tissue was immediately placed in ice-cold isolation buffer [22] and minced with precooled scissors followed by an enzymatic digestion with protease (Subtilisin A, Sigma Aldrich, St Louis, MO, USA). Muscle pieces were then centrifuged at $8,500 \mathrm{~g}$ for $5 \mathrm{~min}$ at $4^{\circ} \mathrm{C}$. After removal of the supernatant fraction, the pellet was resuspended in isolation buffer and homogenised in a glass potter tube. The homogenate was transferred to a centrifuge tube and centrifuged at $1,000 \mathrm{~g}$ for $10 \mathrm{~min}$ at $4^{\circ} \mathrm{C}$. The resulting supernatant fraction was filtered through cheesecloth, and centrifuged at $10,000 \mathrm{~g}$ for $10 \mathrm{~min}$ at $4^{\circ} \mathrm{C}$. The resulting mitochondrial pellet was resuspended in $20 \mu \mathrm{l}$ isolation buffer, and protein content was determined by Fluram assay [28]. Subsequently, highresolution respirometry on a 2-chamber Oxygraph (Oroboros Instruments, Innsbruck, Austria) with automatic palmitate titration was performed, as described previously [22]. Successful mitochondrial respiration measurements were achieved in 11 participants (six males and five females; baseline and fasted assays successful).

Blood analysis Plasma concentrations of glucose, free glycerol, total glycerol and NEFAs were determined on a Cobas FARA centrifugal spectrophotometer (Roche Diagnostics, Basel, Switzerland), as described previously [26]. Triacylglycerol levels were calculated as the difference between total and free glycerol. Serum insulin was analysed with a Human Insulin Specific RIA kit (Millipore, Billerica, MA, USA) on a gamma counter (2470 Automatic Counter Wizard $^{2}$, PerkinElmer, Waltham, MA, USA). Plasma catecholamines were analysed on HPLC and by fluorimetric detection [29]. Serum thyroid-stimulating hormone
(TSH) and free thyroxine (FT4) were analysed as described previously [26].

Statistical analysis Statistical analyses were performed with SPSS Statistics 20.0 for MAC (IBM, Armonk, NY, USA). Reported data are expressed as means $\pm \mathrm{SD}$, unless stated otherwise. A Shapiro-Wilk test was used to test for normal distribution of relevant variables and non-parametric tests were used when appropriate. Two-sided paired sample $t$ tests were used to compare findings between baseline and fasted conditions and between thermoneutral and mild cold conditions. Two-sided independent sample $t$ tests were used to compare findings between men and women. Pearson correlations were used to identify correlations between variables. The level of statistical significance was set at $p<0.05$.

Study approval The Ethics Committee of Maastricht University Medical Centre+ approved the study protocol and all participants provided written informed consent. Procedures were conducted according to the principles of the Declaration of Helsinki.

\section{Results}

Effect offasting on plasma variables NEFA levels were markedly higher after fasting compared with the baseline condition $(1,172 \pm 284$ vs $600 \pm 221 \mu \mathrm{mol} / 1$, respectively; $p<0.001$; Table 1$)$, and plasma glucose $(5.0 \pm 0.3$ vs $3.9 \pm 0.5 \mathrm{mmol} / \mathrm{l}$, baseline vs fasted, respectively; $p<0.001)$ and insulin $(51.4 \pm$ 22.9 vs $38.9 \pm 16.7 \mathrm{pmol} / 1$, baseline vs fasted, respectively; $p=$ $0.066)$ were both decreased upon fasting. Norepinephrine concentrations were similar $(0.97 \pm 0.45$ vs $0.95 \pm 0.40 \mathrm{nmol} /$

Table 1 Plasma hormone and metabolite concentrations at thermoneutral temperature and during mild cold exposure, in the baseline vs fasted condition

\begin{tabular}{|c|c|c|c|c|}
\hline \multirow[t]{2}{*}{ Variable } & \multicolumn{2}{|l|}{ Baseline } & \multicolumn{2}{|l|}{ Fasted } \\
\hline & Thermoneutral & Mild cold & Thermoneutral & Mild cold \\
\hline NEFA $(\mu \mathrm{mol} / 1)$ & $600 \pm 221$ & $815 \pm 200^{\dagger}$ & $1,172 \pm 284^{* * *}$ & $1,588 \pm 529 * * *, \dagger$ \\
\hline Glucose (mmol/l) & $5.0 \pm 0.3$ & $5.0 \pm 0.3$ & $3.9 \pm 0.5^{* * *}$ & $3.9 \pm 0.5 * * *$ \\
\hline Insulin (pmol/l) & $51.4 \pm 22.9$ & $56.3 \pm 20.1$ & $38.9 \pm 16.7$ & $44.4 \pm 11.8$ \\
\hline Norepinephrine (nmol/l) & $0.97 \pm 0.45$ & $2.52 \pm 1.18^{\dagger \dagger}$ & $0.95 \pm 0.40$ & $3.57 \pm 2.11^{\dagger \dagger}$ \\
\hline Epinephrine (nmol/l) & $0.18 \pm 0.11$ & $0.19 \pm 0.09$ & $0.25 \pm 0.15$ & $0.35 \pm 0.23^{\dagger}$ \\
\hline TSH (mU/l) & $2.0 \pm 1.0$ & $1.9 \pm 1.1^{\dagger}$ & $1.1 \pm 0.6^{* *}$ & $1.3 \pm 0.7$ \\
\hline Free T4 (pmol/l) & $14.5 \pm 1.5$ & $14.6 \pm 1.8$ & $14.1 \pm 2.2$ & $14.7 \pm 2.2^{\dagger}$ \\
\hline
\end{tabular}

${ }^{* *} p<0.01$, baseline vs fasted

$* * * p<0.001$, baseline vs fasted

${ }^{\dagger} p<0.05$, mild cold vs thermoneutral

${ }^{\dagger \dagger} p<0.01$, mild cold vs thermoneutral 
1 , baseline vs fasted, respectively; $p=0.90$ ), while epinephrine concentrations tended to be higher upon fasting $(0.18 \pm 0.11 \mathrm{vs}$ $0.25 \pm 0.15 \mathrm{nmol} / \mathrm{l}$, baseline vs fasted respectively; $p=0.07$ ).

Changes in energy expenditure upon fasting Energy expenditure was significantly increased upon mild cold exposure in both the baseline (from $4.6 \pm 0.6$ to $5.2 \pm 0.9 \mathrm{~kJ} / \mathrm{min}, p<0.01$ ) and the fasted condition (from $4.9 \pm 0.8$ to $5.2 \pm 0.9 \mathrm{~kJ} / \mathrm{min}$, $p<0.01)$. NST, expressed as percentage increase in energy expenditure upon cold exposure, was significantly lower in the fasted condition $(12.7 \pm 9.1 \%$ vs $6.7 \pm 5.1 \%, p<0.01$; Table 2 and Fig. 2d). In addition, the absolute increase in energy expenditure upon cold exposure was significantly lower upon fasting $(0.6 \pm 0.5 \mathrm{vs} 0.3 \pm 0.3 \mathrm{~kJ} / \mathrm{min}$, baseline vs fasted respectively; $p<0.05)$. However, since BMR showed a slight increase upon fasting $(\sim 6 \%, p<0.01)$, total whole-body energy expenditure during cold exposure was similar between fasting and baseline conditions $(p>0.05)$.

The respiratory quotient $(\mathrm{RQ})$ was significantly lower upon fasting (Table 2). Cold exposure elicited no significant change in RQ or plasma glucose levels in either condition, while it increased plasma NEFAs in both conditions (Table 1).

Decreased BAT glucose uptake upon fasting In the baseline condition, cold-induced BAT activity was detected in 15 out of 16 participants. Average $\left[{ }^{18} \mathrm{~F}\right] \mathrm{FDG}$ uptake (SUV mean) of all upper body BAT depots was $2.3 \pm 0.8$ and maximal $\left[{ }^{18} \mathrm{~F}\right] \mathrm{FDG}$ uptake (SUV max) in BAT was $10.6 \pm 6.5$. There were no significant differences in BAT glucose uptake between males and females, which is in accordance with previous findings $[22,30]$. Outdoor temperature did not affect cold-stimulated BAT activity. We could detect BAT activity upon fasting in 14 out of the 15 participants that also showed BAT activity at baseline. However, fasting resulted in a marked decrease in cold-induced glucose uptake in BAT: average BAT $\left[{ }^{18} \mathrm{~F}\right] \mathrm{FDG}$ uptake decreased to $1.7 \pm 0.7 \mathrm{SUV}$ and maximal $\left[{ }^{18} \mathrm{~F}\right] \mathrm{FDG}$ uptake decreased to $4.7 \pm 4.4$ SUV (both $p<0.01$ compared with baseline condition; Table 2). Analysis of dynamic PET/ CT imaging of the supraclavicular region revealed similar results. The average supraclavicular BAT glucose uptake rate of all participants was $6.0 \pm 3.4 \mu \mathrm{mol} \mathrm{min}{ }^{-1} 100 \mathrm{~g}^{-1}$ in the baseline condition, and decreased to $2.7 \pm 1.7 \mu \mathrm{mol} \mathrm{min}$ $100 \mathrm{~g}^{-1}$ upon fasting ( $p<0.001$, Fig. 2a-c).

To investigate glucose fluxes into BAT upon fasting in more detail, two-tissue compartment modelling of the dynamic PET/CT data was employed (ESM Fig. 1). $K_{1}$ values, representing the $\left[{ }^{18} \mathrm{~F}\right] \mathrm{FDG}$ influx rate constant from plasma into BAT (including both interstitial and intracellular space), were not different between the baseline and fasted condition $\left(0.09 \pm 0.04\right.$ vs $0.11 \pm 0.03 \mathrm{ml} \mathrm{min}^{-1} \mathrm{ml}^{-1}$, respectively, $p=$ 0.146; Table 3). In addition, the distribution volume (DV) of free (nonphosphorylated) $\left[{ }^{18} \mathrm{~F}\right] \mathrm{FDG}$ in BAT was not different between the baseline and fasted condition $(0.32 \pm 0.13$ vs $0.26 \pm 0.09 \mathrm{ml} / \mathrm{ml}$, respectively, $p=0.185$ ). Interestingly, phosphorylation fraction value, reflecting the proportion of $\left[{ }^{18} \mathrm{~F}\right] \mathrm{FDG}$ that enters the tissue (including both interstitial

Table 2 BAT activity and energy expenditure during baseline and fasted conditions

\begin{tabular}{|c|c|c|c|c|c|c|}
\hline \multirow[t]{2}{*}{ Variable } & \multicolumn{2}{|c|}{ Group values $(n=16)$} & \multicolumn{2}{|c|}{ Males $(n=8)$} & \multicolumn{2}{|c|}{ Females $(n=8)$} \\
\hline & Baseline & Fasted & Baseline & Fasted & Baseline & Fasted \\
\hline$\left[{ }^{18} \mathrm{~F}\right] \mathrm{FDG}$ uptake in BAT (SUV mean) & $2.3 \pm 0.8$ & $1.7 \pm 0.7 * *$ & $2.6 \pm 0.6$ & $1.8 \pm 0.8^{*}$ & $2.1 \pm 0.9$ & $1.7 \pm 0.7 *$ \\
\hline$\left[{ }^{18} \mathrm{~F}\right] \mathrm{FDG}$ uptake in BAT (SUV max) & $10.6 \pm 6.5$ & $4.7 \pm 4.4^{* *}$ & $12.5 \pm 7.6$ & $5.5 \pm 5.8$ & $8.7 \pm 5.0$ & $3.9 \pm 2.5^{*}$ \\
\hline Glucose uptake rate in BAT $\left(\mu \mathrm{mol} \min ^{-1} 100 \mathrm{~g}^{-1}\right)$ & $6.0 \pm 3.4$ & $2.7 \pm 1.7^{* *}$ & $7.3 \pm 3.8$ & $2.8 \pm 2.1^{*}$ & $4.8 \pm 2.5$ & $2.5 \pm 1.4^{*}$ \\
\hline \multicolumn{7}{|l|}{ Fixed volume analysis } \\
\hline$\left[{ }^{18} \mathrm{~F}\right] \mathrm{FDG}$ uptake in BAT (SUV mean) & $7.4 \pm 5.0$ & $3.0 \pm 2.6^{* *}$ & $8.7 \pm 6.1$ & $3.3 \pm 3.4$ & $5.9 \pm 3.5$ & $2.6 \pm 1.6^{*}$ \\
\hline 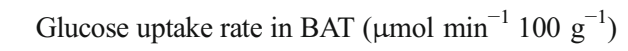 & $17.3 \pm 14.3$ & $4.8 \pm 5.4^{* *}$ & $20.4 \pm 17.8$ & $4.8 \pm 7.2$ & $10.3 \pm 6.9$ & $3.8 \pm 3.2 *$ \\
\hline \multicolumn{7}{|l|}{ Energy expenditure (kJ/min) } \\
\hline Thermoneutral & $4.6 \pm 0.6$ & $4.9 \pm 0.8^{* *}$ & $5.1 \pm 0.5$ & $5.5 \pm 0.5^{* *}$ & $4.2 \pm 0.3^{\ddagger}$ & $4.3 \pm 0.3 \ddagger$ \\
\hline Mild cold & $5.2 \pm 0.9^{\dagger}$ & $5.2 \pm 0.9^{\dagger}$ & $6.0 \pm 0.6^{\dagger}$ & $6.0 \pm 0.5^{\dagger}$ & $4.5 \pm 0.4^{\dagger, \dagger}$ & $4.5 \pm 0.4^{\dagger, \ddagger}$ \\
\hline$\%$ NST & $12.7 \pm 9.1$ & $6.7 \pm 5.1 * *$ & $14.8 \pm 12.5$ & $10.2 \pm 5.1 *$ & $7.9 \pm 5.6$ & $4.6 \pm 4.8$ \\
\hline \multicolumn{7}{|l|}{ RQ } \\
\hline Thermoneutral & $0.80 \pm 0.04$ & $0.74 \pm 0.03 * *$ & $0.82 \pm 0.05$ & $0.75 \pm 0.04 *$ & $0.80 \pm 0.04$ & $0.75 \pm 0.03 * *$ \\
\hline Mild cold & $0.81 \pm 0.04$ & $0.76 \pm 0.04 * *$ & $0.81 \pm 0.04$ & $0.76 \pm 0.04^{*}$ & $0.81 \pm 0.03$ & $0.78 \pm 0.05^{*}$ \\
\hline
\end{tabular}

${ }^{*} p<0.05$, baseline vs fasted

$* * p<0.01$, baseline vs fasted

${ }^{\dagger} p<0.05$, mild cold vs thermoneutral

${ }^{*} p<0.05$ males vs females 
a

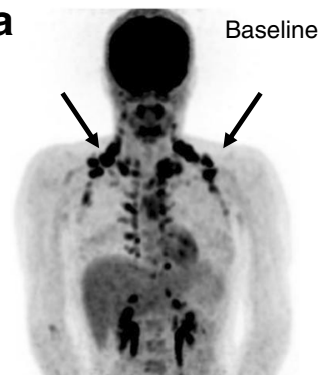

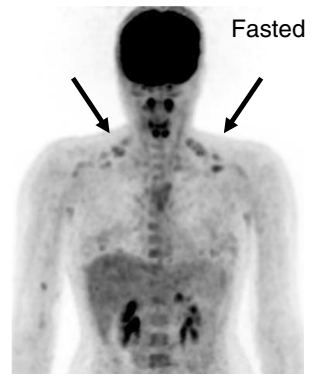

C
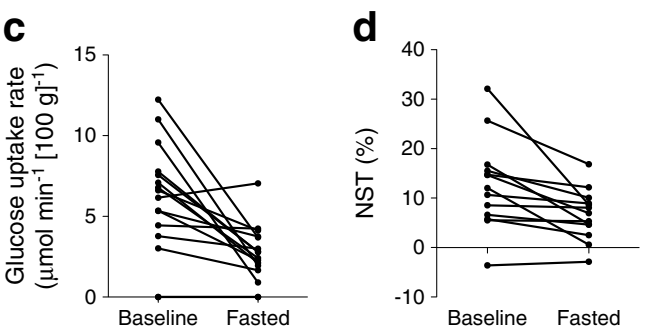

e

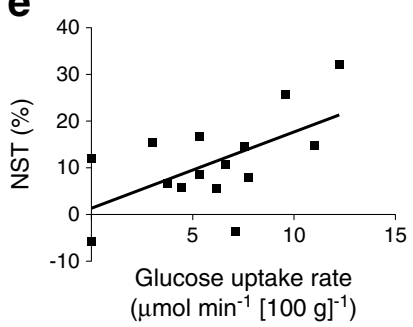

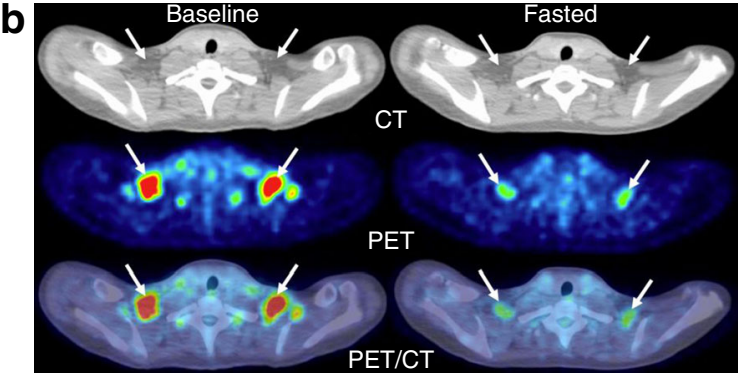

f

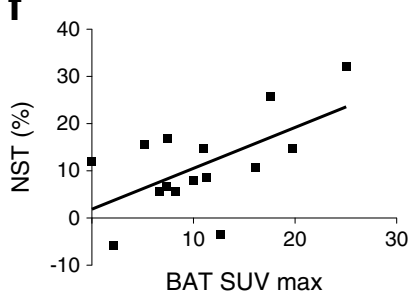

Fig. 2 BAT glucose uptake rate and NST are significantly decreased upon fasting. A significant association between BAT activity and NST is found in the baseline condition. (a) $\left[{ }^{18} \mathrm{~F}\right] \mathrm{FDG}-\mathrm{PET}$ images after cold exposure in a female participant in the baseline (left) and fasted (right) condition. Black arrows indicate supraclavicular BAT activity. (b) Transversal CT, PET and PET/CT fusion slices (as indicated) of the

and intracellular space) that is eventually metabolised $\left(k_{3} /\right.$ $\left.\left[k_{2}+k_{3}\right]\right)$, was significantly lower upon fasting $(0.25 \pm 0.11$ vs $0.11 \pm 0.06$, baseline vs fasted, respectively, $p<0.01)$.

BAT glucose uptake rate and NST were significantly correlated in the baseline condition (Fig. 2e), as were maximal $\left[{ }^{18} \mathrm{~F}\right] \mathrm{FDG}$ uptake in BAT and NST (Fig. 2f). Changes in NST and BAT glucose uptake from the baseline to the fasting condition were not related.

Glucose uptake in other tissues In contrast to marked decreases in $\left[{ }^{18} \mathrm{~F}\right] \mathrm{FDG}$ uptake in BAT, no changes in $\left[{ }^{18} \mathrm{~F}\right] \mathrm{FDG}$ uptake during cold stimulation were observed in skeletal muscle, visceral WAT, liver and brain in the fasted compared with the baseline condition (Fig. 3). Subcutaneous WAT showed a small but significant increase in cold-

Table 3 Rate constants for two-tissue compartment modelling of $\left[{ }^{18} \mathrm{~F}\right] \mathrm{FDG}$ metabolism in BAT

\begin{tabular}{lll}
\hline & Baseline & Fasted \\
\hline$K_{1}, \mathrm{ml} \mathrm{min}^{-1} \mathrm{ml}^{-1}$ & $0.09 \pm 0.04$ & $0.11 \pm 0.03$ \\
$k_{2}, \mathrm{~min}^{-1}$ & $0.33 \pm 0.17$ & $0.45 \pm 0.13$ \\
$k_{3}, \mathrm{~min}^{-1}$ & $0.10 \pm 0.06$ & $0.05 \pm 0.02^{* *}$ \\
$\quad \mathrm{DV}$ of free $\left[{ }^{18} \mathrm{~F}\right] \mathrm{FDG}\left(K_{1} / k_{2}\right)$, & $0.32 \pm 0.13$ & $0.26 \pm 0.09$ \\
$\quad$ ml plasma/ml tissue & & \\
Phosphorylation fraction $\left[k_{3} /\left(k_{2}+k_{3}\right)\right]$ & $0.25 \pm 0.11$ & $0.11 \pm 0.06^{* *}$ \\
\hline
\end{tabular}

${ }^{* *} p<0.01$, baseline vs fasted, $n=14$

DV, distribution volume supraclavicular region showing $\left[{ }^{18} \mathrm{~F}\right] \mathrm{FDG}$ uptake in BAT locations (white arrows) after cold exposure in the baseline and the fasted condition. (c, d) Individual data on BAT glucose uptake rate and NST (\%). (e, f) Associations between NST $(\%)$ and BAT glucose uptake rate $\left(r^{2}=0.347\right.$, $p<0.05$ ), and between NST (\%) and maximal BAT activity (BAT SUV $\left.\max ; r^{2}=0.356, p<0.05\right)$ in the baseline condition

stimulated glucose uptake upon fasting $(0.21 \pm 0.07$ to $0.26 \pm$ 0.09 SUV mean, $p<0.05$ ).

Skeletal muscle mitochondrial respiration Next to BAT, skeletal muscle is a putative alternative contributor to NST via mitochondrial proton leak, resulting in respiration that is not coupled to ATP production [31]. Analysis of mitochondrial oxygen consumption using isolated mitochondria revealed no differences in oligomycin-insensitive (state 4o) respiration, reflecting basal proton leak, between the baseline and fasted samples $\left(175.0 \pm 68.7 \mathrm{vs} 164.5 \pm 63.1 \mathrm{pmol} \mathrm{O}_{2} \mathrm{~s}^{-1} \mathrm{mg}^{-1}\right.$ mitochondrial protein, respectively, $p>0.05$; ESM Fig. 2a), indicating no contribution of skeletal muscle mitochondrial proton leak to the decrease in NST upon fasting. Fatty acids stimulated the oligomycin-insensitive (state 40 ) respiration in a

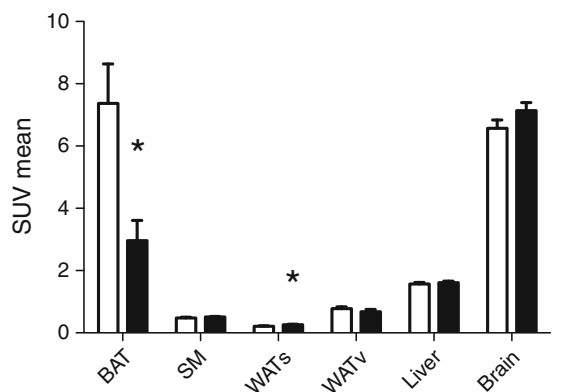

Fig. 3 Mean ( \pm SEM) uptake of $\left[{ }^{18}\right.$ F]FDG in BAT and other tissues during the baseline (white bars) and fasted (black bars) conditions. ${ }^{*} p<0.05$ fasted vs baseline. SM, skeletal muscle; WATs, subcutaneous white adipose tissue; WATv, visceral white adipose tissue. $n=16$ (for brain $n=9$ ) 
Table 4 Body temperatures and skin perfusion at thermoneutral temperature and during mild cold exposure, in the baseline vs fasted condition

\begin{tabular}{|c|c|c|c|c|c|c|}
\hline \multirow[t]{2}{*}{ Variable } & \multicolumn{3}{|l|}{ Baseline } & \multicolumn{3}{|l|}{ Fasted } \\
\hline & Thermoneutral & Mild cold & Delta & Thermoneutral & Mild cold & Delta \\
\hline Core temperature $\left({ }^{\circ} \mathrm{C}\right)$ & $37.06 \pm 0.26$ & $37.10 \pm 0.24$ & $0.04 \pm 0.24$ & $37.22 \pm 0.28^{* *}$ & $36.96 \pm 0.29 *, \dagger \dagger$ & $-0.26 \pm 0.21 * *$ \\
\hline Mean skin temperature $\left({ }^{\circ} \mathrm{C}\right)$ & $35.4 \pm 0.2$ & $31.4 \pm 0.8^{\dagger \dagger}$ & $-3.9 \pm 0.9$ & $35.5 \pm 0.3$ & $31.6 \pm 1.0^{\dagger \dagger}$ & $-3.9 \pm 1.0$ \\
\hline Skin perfusion hand relative $\%$ & 100 & $14.6 \pm 10.0^{\dagger \dagger}$ & $-85.4 \pm 10.0$ & 100 & $15.3 \pm 11.6^{\dagger \dagger}$ & $-84.7 \pm 11.6$ \\
\hline Skin perfusion abdomen relative $\%$ & 100 & $76.0 \pm 21.4^{\dagger \dagger}$ & $-24.0 \pm 21.4$ & 100 & $67.1 \pm 15.2^{\dagger \dagger}$ & $-32.9 \pm 15.2$ \\
\hline
\end{tabular}

$* p<0.05$, baseline vs fasted

$* * p<0.01$, baseline vs fasted

${ }^{\dagger} p<0.01$, mild cold vs thermoneutral

dose-dependent manner. However, calculated half-maximal effective concentration $\left(\mathrm{EC}_{50}\right)$ values $(278 \pm 236$ vs $235 \pm$ $194 \mathrm{nmol} / \mathrm{l}$, baseline vs fasted, respectively, $p>0.05$; ESM Fig. 2b) and maximal fatty acid-induced uncoupled respiration values (Vmax; 2,486 \pm 690 vs $2,082 \pm 818$ pmol $\mathrm{O}_{2} \mathrm{~s}^{-1} \mathrm{mg}^{-1}$, baseline vs fasted, respectively, $p>0.05$; ESM Fig. 2c) were similar in both conditions, indicating comparable sensitivity to the uncoupling effects of fatty acids in the baseline and fasted condition.

Core and skin temperatures and skin perfusion Temperature of the water-perfused suit showed a similar decrease during the cooling protocol in the baseline (from $30.3 \pm 0.6^{\circ} \mathrm{C}$ to $25.4 \pm$ $0.8^{\circ} \mathrm{C}$ ) and the fasted condition (from $30.3 \pm 0.5^{\circ} \mathrm{C}$ to $25.6 \pm$ $0.9^{\circ} \mathrm{C}$ ). Consequently, the decrease in mean skin temperature during the cooling protocol was also similar between conditions $\left(-3.9 \pm 0.9^{\circ} \mathrm{C}\right.$ vs $-3.9 \pm 1.0^{\circ} \mathrm{C}, p=0.628$, respectively; Table 4). In addition, skin perfusion of the hand and abdomen were not different between the conditions. Interestingly, core temperature showed a significant decrease upon cold exposure in the fasted condition $\left(-0.26 \pm 0.21^{\circ} \mathrm{C}, p<0.001\right)$, in contrast to an unchanged core temperature in the baseline condition $\left(0.04 \pm 0.24^{\circ} \mathrm{C}, p=0.557\right)$. The difference in cold-induced core temperature between the fasted and the baseline condition was significantly correlated to the difference in BAT glucose uptake rate $\left(r^{2}=0.350, p<0.05\right.$; Fig. 4$)$ and BAT phosphorylation fraction $\left(r^{2}=0.308, p<0.05\right)$ between these conditions.

\section{Discussion}

BAT has been proposed as a target to stimulate glucose disposal under insulin-resistant conditions, such as type 2 diabetes. When peripheral tissues are resistant for the effect of insulin to stimulate glucose uptake, this leads to impaired insulin-induced glucose clearance. However, even under such insulin-resistant conditions, glucose uptake in peripheral tissues can still be increased via non-insulin dependent pathways, such as the AMPK pathway that can be activated by exercise or by pharmacological means [32]. Whether coldinduced activation of BAT could also be a target to improve glucose clearance under insulin-resistant conditions in humans is so far unknown. Here we used prolonged fasting as a model to induce insulin resistance, and examined whether cold stimulation could still activate glucose uptake into BAT under such insulin-resistant conditions. Prolonged fasting is an interesting model, as peripheral insulin resistance develops to preserve glucose as a substrate for the brain; however, even under hyperinsulinaemic-euglycaemic clamp conditions, when glucose availability is ample, peripheral glucose uptake is still reduced after $60 \mathrm{~h}$ of fasting, as we have shown recently [18]. Although we were not able to repeat measurements of whole-body insulin resistance here, we followed a very similar protocol as in our previous publication and levels of plasma NEFAs, glucose and insulin are in agreement with this previous study, convincing us that participants were indeed more insulin-resistant upon fasting in the present study. We now show that cold-induced glucose uptake into BAT is markedly reduced after prolonged fasting. If these results also apply to other insulin-resistant conditions, it may suggest that isolated cold therapy may not be able to improve glucose homeostasis via glucose clearance by BAT under such conditions.

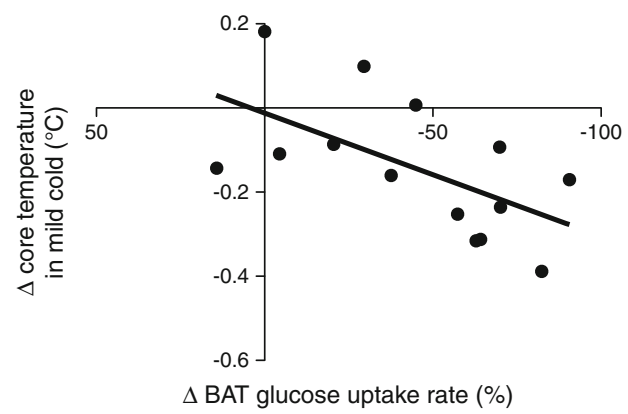

Fig. 4 Relationship between the difference in core temperature during cold exposure between the fasted and the baseline condition ( $\Delta$ core temperature in mild cold) and the difference in BAT glucose uptake rate between these conditions ( $\Delta$ glucose uptake rate); $r^{2}=0.350, p<0.05$ 
The dynamic PET scanning protocol allowed us to investigate disturbances in cold-induced BAT glucose uptake upon prolonged fasting in more detail, using two-tissue compartment modelling. Theoretically, decreased glucose uptake rates into BAT could be either due to decreased glucose availability to the tissue (e.g. due to decreased blood flow, decreased plasma glucose or decreased glucose extraction) and/or due to decreased cellular glucose uptake rate. Interestingly, we found no significant changes in $\left[{ }^{18} \mathrm{~F}\right] \mathrm{FDG}$ influx rates $\left(K_{l}\right)$ and $\left[{ }^{18} \mathrm{~F}\right] \mathrm{FDG}$ distribution volume in BAT upon fasting, indicating that the decrease in BAT glucose uptake rate was not due to a lower supply of glucose towards BAT, i.e. glucose transport to BAT interstitial space was not hampered. Rather, the model indicates a lower cellular uptake and/or decreased phosphorylation rate of glucose, as indicated by the lower phosphorylation fraction in the fasted condition. As stated above, it should be noted that glucose transport into BAT reflects movement of $\left[{ }^{18} \mathrm{~F}\right] \mathrm{FDG}$ to both intracellular and interstitial space [33] and that two-tissue compartment modelling does not completely discriminate between (GLUTmediated) glucose transport into brown adipocytes and/or subsequent phosphorylation by hexokinase. We are therefore not able to distinguish between brown adipocyte $\left[{ }^{18} \mathrm{~F}\right] \mathrm{FDG}$ uptake or $\left[{ }^{18} \mathrm{~F}\right] \mathrm{FDG}$ phosphorylation as the main determinant of decreased BAT $\left[{ }^{18} \mathrm{~F}\right] \mathrm{FDG}$ uptake upon fasting. As in other insulin-resistant conditions both of these processes are disturbed as well [34]; this points towards comparable mechanisms at play in BAT during fasting-induced insulin-resistant conditions.

Interestingly, the decrease in cold-stimulated glucose uptake after fasting is specific for BAT, since this was not observed in other tissues. In subcutaneous WAT, $\left[{ }^{18} \mathrm{~F}\right] \mathrm{FDG}$ uptake was increased, which may be explained by an increased triacylglycerol/fatty acid cycling, whereby $\alpha$ glycerol phosphate, which provides the backbone for esterification of NEFAs, is derived from plasma glucose uptake, as demonstrated previously [35]. The energy required for this process might actually contribute to the increase in energy expenditure that we measured upon fasting [36]. In skeletal muscle, cold-induced glucose uptake was not affected by fasting; these results may indicate that even though cold exposure may not be able to increase glucose clearance under insulin-resistant conditions via BAT, the effect of cold exposure on other glucose-consuming tissues may still be worth exploring as a therapy to boost glucose clearance under insulin-resistant states.

Consistent with previous publications [37, 38], we found that BMR was increased upon fasting, most likely due to increased whole-body sympathetic activity, as indicated by the elevated epinephrine levels. Furthermore, we found that NST was decreased upon fasting, which can be partly explained by higher BMR, and we found a correlation between BAT glucose uptake rate and NST at baseline, but not in the fasted state. At first instance, this latter finding could argue against a direct relationship between BAT thermogenic activity and whole-body energy expenditure. However, it should be noted that $\left[{ }^{18} \mathrm{~F}\right] \mathrm{FDG}$ uptake is not a direct measure of the thermogenic activity of BAT. Although a relationship between $\left[{ }^{18} \mathrm{~F}\right] \mathrm{FDG}$ uptake and thermogenesis in BAT will apply under most conditions, a shift in substrate oxidation towards fatty acid metabolism during fasting might result in increased reliance on fatty acid oxidation for BAT's heat production. Thus, using only glucose uptake as a measure of BAT activity could possibly lead to an underestimation of true BAT thermogenesis under fasted conditions. Future studies using alternative tracers, such as $\left[{ }^{18} \mathrm{~F}\right] \mathrm{THA}$ (as a measure of NEFA uptake) and ${ }^{11} \mathrm{C}$-acetate (as a measure of oxidative metabolism), are needed to determine the thermogenic activity of BAT under prolonged fasting conditions. Nevertheless, it is interesting to note that we did find that the fasting-induced decrease in BAT glucose uptake was related to a decrease in core temperature upon cold exposure. Based on our data, it is therefore tempting to speculate that, although a reduction in coldstimulated glucose uptake into BAT upon fasting may be beneficial to spare glucose availability for the brain, an impaired capacity to regulate body temperature may be the consequence. To the best of our knowledge, this is the first study that shows a direct link between changes in BAT glucose uptake and core temperature, indicating a role for BAT in maintaining core temperature upon cold exposure in humans.

In conclusion, this study shows that under prolonged fasting-induced insulin-resistant conditions, cold-stimulated glucose uptake into BAT is reduced, due to a reduced cellular glucose uptake, and is accompanied with reduced NST. Furthermore, our data indicate that the decrease in coldstimulated BAT glucose uptake upon fasting is related to a decrease in core temperature during cold exposure, suggesting a role for BAT in maintaining a constant core temperature in cold environment. If the observed reduction in the capacity of BAT to take up glucose upon cold exposure is also applicable to other insulin-resistant conditions, such as type 2 diabetes, our data may indicate that a focus on cold-induced stimulation of BAT alone may not be a valid way to improve glucose clearance under such conditions. However, follow-up studies are needed to examine whether glucose uptake into BAT can be activated by other means under insulinresistant conditions. Specifically, studies in type 2 diabetic patients are urgently awaited.

Acknowledgements We thank M. Visser, K. Jansen, R. Hensgens and E. Broeders (Maastricht University Medical Centre+) for assistance during the experiments and M. Bauwens, I. Pooters, J. Stegen, N. Hendrix and P. Menheere (Maastricht University Medical Centre+) for assistance with the biochemical analyses. The technical support of P. Schoffelen, L. Wouters and E. Beijer (Maastricht University Medical Centre+) was highly appreciated. Finally, we thank our TherMU and TherNU research group members for fruitful discussions. 
Funding This work was supported by the EU FP7 project DIABAT (HEALTH-F2-2011-278373 to WDvML) and by the Netherlands Organization for Scientific Research (TOP 91209037 to WDvML). Trial registration number: NTR3523.

Duality of interest The authors declare that there is no duality of interest associated with this manuscript.

Contribution statement MJWH was responsible for study design, data acquisition and data analysis and wrote the manuscript. RW and BB contributed to the data analysis. JH and AG contributed to the data acquisition and data analysis. FMM, PS and WDvML contributed to the study design and interpretation of data. All authors contributed to the critical revision of the manuscript and approved the final version. MJWH is the guarantor of this work and, as such, has full access to all the data in the study and takes responsibility for the integrity of the data and the accuracy of the data analysis.

\section{References}

1. Harms M, Seale P (2013) Brown and beige fat: development, function and therapeutic potential. Nat Med 19:1252-1263

2. Schulz TJ, Tseng YH (2013) Brown adipose tissue: development, metabolism and beyond. Biochem J 453:167-178

3. van Marken Lichtenbelt WD, Vanhommerig JW, Smulders NM et al (2009) Cold-activated brown adipose tissue in healthy men. N Engl J Med 360:1500-1508

4. Virtanen KA, Lidell ME, Orava J et al (2009) Functional brown adipose tissue in healthy adults. N Engl J Med 360:1518-1525

5. Ouellet V, Labbe SM, Blondin DP et al (2012) Brown adipose tissue oxidative metabolism contributes to energy expenditure during acute cold exposure in humans. J Clin Invest 122:545-552

6. Trayhurn P (1979) Thermoregulation in the diabetic-obese $(\mathrm{db} / \mathrm{db})$ mouse. The role of non-shivering thermogenesis in energy balance. Pflugers Arch 380:227-232

7. Matz JM, LaVoi KP, Epstein PN, Blake MJ (1996) Thermoregulatory and heat-shock protein response deficits in cold-exposed diabetic mice. Am J Physiol 270:R525-R532

8. Yoshioka K, Yoshida T, Wakabayashi Y, Nishioka H, Kondo M (1989) The role of insulin in norepinephrine turnover and thermogenesis in brown adipose tissue after acute cold-exposure. Endocrinol Jpn 36:491-499

9. Mercer SW, Trayhurn P (1984) The development of insulin resistance in brown adipose tissue may impair the acute cold-induced activation of thermogenesis in genetically obese (ob/ob) mice. Biosci Rep 4: 933-940

10. Marette A, Deshaies Y, Collet AJ, Tulp O, Bukowiecki LJ (1991) Major thermogenic defect associated with insulin resistance in brown adipose tissue of obese diabetic SHR/N-cp rats. Am J Physiol 261: E204-E213

11. Burcelin R, Kande J, Ricquier D, Girard J (1993) Changes in uncoupling protein and GLUT4 glucose transporter expressions in interscapular brown adipose tissue of diabetic rats: relative roles of hyperglycaemia and hypoinsulinaemia. Biochem J 291(Pt 1): 109-113

12. Saito M, Okamatsu-Ogura Y, Matsushita M et al (2009) High incidence of metabolically active brown adipose tissue in healthy adult humans: effects of cold exposure and adiposity. Diabetes 58:1526-1531

13. Cypess AM, Lehman S, Williams G et al (2009) Identification and importance of brown adipose tissue in adult humans. N Engl J Med 360:1509-1517
14. Yoneshiro T, Aita S, Matsushita M et al (2011) Age-related decrease in cold-activated brown adipose tissue and accumulation of body fat in healthy humans. Obesity 19:1755-1760

15. Ouellet V, Routhier-Labadie A, Bellemare W et al (2011) Outdoor temperature, age, sex, body mass index, and diabetic status determine the prevalence, mass, and glucose-uptake activity of $18 \mathrm{~F}-\mathrm{FDG}$-detected BAT in humans. J Clin Endocrinol Metab 96:192-199

16. Lean ME, Murgatroyd PR, Rothnie I, Reid IW, Harvey R (1988) Metabolic and thyroidal responses to mild cold are abnormal in obese diabetic women. Clin Endocrinol (Oxf) 28:665-673

17. Bergman BC, Cornier MA, Horton TJ, Bessesen DH (2007) Effects of fasting on insulin action and glucose kinetics in lean and obese men and women. Am J Physiol Endocrinol Metab 293:E1103-E1111

18. Hoeks J, van Herpen NA, Mensink M et al (2010) Prolonged fasting identifies skeletal muscle mitochondrial dysfunction as consequence rather than cause of human insulin resistance. Diabetes 59:2117-2125

19. Salgin B, Marcovecchio ML, Humphreys SM et al (2009) Effects of prolonged fasting and sustained lipolysis on insulin secretion and insulin sensitivity in normal subjects. Am J Physiol Endocrinol Metab 296:E454-E461

20. DeFronzo RA, Tripathy D (2009) Skeletal muscle insulin resistance is the primary defect in type 2 diabetes. Diabetes Care 32(Suppl 2): S157-S163

21. Cannon B, Nedergaard J (2010) Metabolic consequences of the presence or absence of the thermogenic capacity of brown adipose tissue in mice (and probably in humans). Int $\mathrm{J}$ Obes 34(Suppl 1):S7-S16

22. van der Lans AA, Hoeks J, Brans B et al (2013) Cold acclimation recruits human brown fat and increases nonshivering thermogenesis. J Clin Invest 123:3395-3403

23. Bergstrom J, Hultman E, Roch-Norlund AE (1972) Muscle glycogen synthetase in normal subjects. Basal values, effect of glycogen depletion by exercise and of a carbohydrate-rich diet following exercise. Scand J Clin Lab Invest 29:231-236

24. van Marken Lichtenbelt WD, Daanen HA, Wouters L et al (2006) Evaluation of wireless determination of skin temperature using iButtons. Physiol Behav 88:489-497

25. Kelley DE, Williams KV, Price JC (1999) Insulin regulation of glucose transport and phosphorylation in skeletal muscle assessed by PET. Am J Physiol 277:E361-E369

26. Vosselman MJ, van der Lans AA, Brans B et al (2012) Systemic beta-adrenergic stimulation of thermogenesis is not accompanied by brown adipose tissue activity in humans. Diabetes 61:3106-3113

27. Vosselman MJ, Brans B, van der Lans AA et al (2013) Brown adipose tissue activity after a high-calorie meal in humans. Am J Clin Nutr 98:57-64

28. Hoeks J, Briede JJ, de Vogel J et al (2008) Mitochondrial function, content and ROS production in rat skeletal muscle: effect of high-fat feeding. FEBS Lett 582:510-516

29. Willemsen JJ, Ross HA, Jacobs MC et al (1995) Highly sensitive and specific HPLC with fluorometric detection for determination of plasma epinephrine and norepinephrine applied to kinetic studies in humans. Clin Chem 41:1455-1460

30. Orava J, Nuutila P, Lidell ME et al (2011) Different metabolic responses of human brown adipose tissue to activation by cold and insulin. Cell Metab 14:272-279

31. Wijers SL, Schrauwen P, Saris WH, van Marken Lichtenbelt WD (2008) Human skeletal muscle mitochondrial uncoupling is associated with cold induced adaptive thermogenesis. PLoS One 3:e1777

32. O'Neill HM (2013) AMPK and exercise: glucose uptake and insulin sensitivity. Diabetes Metab J 37:1-21 
33. Williams KV, Price JC, de Kelley (2001) Interactions of impaired glucose transport and phosphorylation in skeletal muscle insulin resistance: a dose-response assessment using positron emission tomography. Diabetes 50:2069-2079

34. Shulman GI (2000) Cellular mechanisms of insulin resistance. J Clin Invest 106:171-176

35. Wolfe RR, Peters EJ, Klein S, Holland OB, Rosenblatt J, Gary H Jr (1987) Effect of short-term fasting on lipolytic responsiveness in normal and obese human subjects. Am J Physiol 252:E189-E196
36. Weber JM, Reidy SP (2012) Extending food deprivation reverses the short-term lipolytic response to fasting: role of the triacylglycerol/ fatty acid cycle. J Exp Biol 215:1484-1490

37. Mansell PI, Fellows IW, Macdonald IA (1990) Enhanced thermogenic response to epinephrine after 48-h starvation in humans. Am J Physiol 258:R87-R93

38. Zauner C, Schneeweiss B, Kranz A et al (2000) Resting energy expenditure in short-term starvation is increased as a result of an increase in serum norepinephrine. Am J Clin Nutr 71:1511-1515 